Check for updates

Cite this: RSC Adv., 2017, 7, 45624

\title{
Activation of persulfate by MMIOC for highly efficient degradation of rhodamine $\mathrm{B} \uparrow$
}

\begin{abstract}
Huanling Xie iD
Mesoporous magnetic iron oxide composites (MMIOCs) were successfully prepared using one-step evaporation induced self-assembly using an organic ferrocene surface active agent as the iron source, and innovatively studied as a potential alternative to mesoporous $\mathrm{CO}_{3} \mathrm{O}_{4}$ for ammonium persulfate (PS) activation to generate active radicals. MMIOCs were characterized by $\mathrm{X}$-ray diffraction (XRD), X-ray photoelectron spectroscopy (XPS), $N_{2}$ adsorption/desorption, transmission electron microscopy (TEM) and using a vibrating sample magnetometer (VSM). Among the different $\mathrm{Fe}_{x} \mathrm{O}_{y} / \mathrm{PS}$ systems tested, the MMIOC displayed the highest catalytic activity for PS activation, achieving almost complete degradation of $1 \mathrm{mM}$ rhodamine $\mathrm{B}(\mathrm{RhB})$ within 120 minutes at $0.2 \mathrm{~g} \mathrm{~L}^{-1}$ catalyst and $40.0 \mathrm{mM}$ PS. The superior catalytic performance of the MMIOC was ascribed to the combination of iron ions inlayed or embedded in the inner or outer surface, forming catalytically active sites and the magnetic mesoporous structure. Several factors played important roles in controlling the degradation of RhB. The equilibrium between $\equiv$ Fe(II) and $\equiv \mathrm{Fe}$ (II) for PS activation in solution ensured continuous generation of the hydroxyl and sulfate radicals, and the latter made the predominant contribution for RhB degradation. Considering its outstanding catalytic activity, excellent reusability and long-term stability, MMIOC could be an ideal catalyst for the degradation of RhB. These results provide an applied solution for disposing of toxic organic wastewater in a relatively available environment, and a scientific foundation for solving refractory organic contaminant related to environmental management.
\end{abstract}

Received 12th July 2017

Accepted 5th September 2017

DOI: $10.1039 / c 7 r a 07645 b$

rsc.li/rsc-advances
Transition metal ions $\left(\mathrm{Co}^{2+}, \mathrm{Cu}^{2+}, \mathrm{Ni}^{2+}, \mathrm{Fe}^{2+}, \mathrm{Ag}^{+}, \mathrm{Ru}^{3+}\right)$ are used for activation of PS to generate sulfate radicals. It has become a hotspot of research to synthesize transition metal oxides, for instance $\mathrm{Co}_{3} \mathrm{O}_{4}$ loaded on supports, $\mathrm{LDH},{ }^{13}$ graphitic carbon nitride ${ }^{14}$ and ordered mesoporous frameworks. ${ }^{5}$

Magnetic mesoporous composites (MMC) could be prepared using single-step or multi-step method. First, with inorganic ferric iron precursor, MMC is in situ synthesized by one-step hydrothermal method..$^{15}$ Second, mesoporous materials must be prepared to produce mesoporous structure. Then, mesoporous materials are integrated with transition metal oxides via various pathways, such as sol-gel, ${ }^{16-18}$ solvothermal treatment $^{19,20}$ and mixed modification. ${ }^{21}$ Third, Tao group have reported one-pot hydrothermal preparation for MMC with long chain containing hydrophobic ferrocene terminal group as both structure-directing agent and magnetic source. ${ }^{22,23}$ However, for example, Co contents generally leak from the catalysts to the solution, leading to heavy metal pollution and thus require additional recycling operation costs. ${ }^{1,24-26}$

To overcome the above mentioned disadvantages, herein, we selected iron ions, as active component. An improved strategy was reported for the one-step evaporation induced selfassembly synthesis for MMIOC. The key point of this strategy is that iron ions could be effectively scattered and inlaid or embedded in inner or outer surface of magnetic mesoporous
The School of Chemistry and Chemical Engineering, Chongqing University of Technology, Chongqing, China, 400054. E-mail: xhl2007@cqut.edu.cn; Tel: +86 13647638549

$\dagger$ Electronic supplementary information (ESI) available. See DOI: 10.1039/c7ra07645b 
materials, synthesized with ferrocene surfactant as structuredirecting agents, which were applied as heterogeneous activator to degrade the RhB in the presence of PS. The objectives of the present study were to evaluate the degradation performance of MMIOC. Moreover, considerable efforts have been devoted to the synthesis of MMIOC to be free of active component leakage, and to be reused several times. Hence, herein, typical generated radicals in different MMIOC/PS systems are identified, and a reasonable $\mathrm{RhB}$ degradation mechanism is proposed.

\section{Experimental}

\subsection{Reagents and materials}

$N, N$-Dimethylaminomethylferrocene $\left(\mathrm{C}_{13} \mathrm{H}_{17} \mathrm{FeN}\right), \quad$ triblock copolymer P123 $\left(\mathrm{EO}_{20} \mathrm{PO}_{70} \mathrm{EO}_{20}\right)$ and $\mathrm{F} 127\left(\mathrm{EO}_{106} \mathrm{PO}_{70} \mathrm{EO}_{106}\right)$ were purchased from J\&K Chemical Reagent Co. Ltd. 1-Bromohexadecane $\left(\mathrm{C}_{16} \mathrm{H}_{33} \mathrm{Br}\right)$, tetraethoxysilane (TEOS), ethanol, potassium chloride, ammonium ferrous sulfate, 1,3,5-trimethylbenzene $\left(\mathrm{C}_{9} \mathrm{H}_{12}\right)$, hydrazine hydrate $\left(\mathrm{N}_{2} \mathrm{H}_{4} \mathrm{H}_{2} \mathrm{O}\right)$, ferric nitrate $\left(\mathrm{Fe}\left(\mathrm{NO}_{3}\right)_{3} \cdot 9 \mathrm{H}_{2} \mathrm{O}\right)$, ammonia solution $(28 \%)$, ethanol, ammonium persulfate (PS) and $\mathrm{RhB}$ of analytical grades were purchased from Southwest Chemical Reagent Co. Ltd., China. All reagents were used without further purification.

\subsection{Preparation and characterization}

2.2.1 Synthesis of structure-directing agent $\mathrm{FcC}_{16} \mathrm{Br}^{-} \mathrm{N}^{+}$. Equal quantities of $N, N$-dimethylaminomethylferrocene and alkyl bromide were reacted for 2 hours under nitrogen at $60^{\circ} \mathrm{C}$. The product obtained from the reaction was recrystallized with acetone twice. Then, it was dried under vacuum at room temperature.

Yield: $60 \%$; bright yellow crystal.

${ }^{1} \mathrm{H}$ NMR (MeOD): 4.518-4.441 (t, 5H), 4.299 (t, 2H), 4.299 (d, $2 \mathrm{H}), 3.35(\mathrm{~d}, 2 \mathrm{H}), 3.215-3.181(\mathrm{~m}, 2 \mathrm{H}), 2.955(\mathrm{~s}, 6 \mathrm{H}), 1.825-1.81$ (m, 2H), 1.397-1.321 (m, 26H), 0.934-0.907 (m, 2H).

Elemental analysis calcd (\%) for $\mathrm{C}_{29} \mathrm{H}_{50} \mathrm{FeBrN}$ : C 63.52, $\mathrm{H}$ 9.13, N 2.56.

2.2.2 Synthesis of MMIOC with $\mathrm{FcC}_{16} \mathrm{Br}^{-} \mathrm{N}^{+}$as structuredirecting agent. The synthesis of MMIOC was carried out using TEOS as silica source and $\mathrm{FcC}_{16} \mathrm{Br}^{-} \mathrm{N}^{+}$as structuredirecting agent in neutral medium through an evaporation induced self-assembly and hydrothermal method. In a typical synthesis, $\mathrm{FcC}_{16} \mathrm{Br}^{-} \mathrm{N}^{+}$was dissolved in ethanol with a small amount of $\mathrm{KCl}(0.1 \mathrm{M})$ under stirring to obtain a homogeneous mixture. Then, TEOS was added to the mixture at room temperature. The molar composition of the resulting mixture was 1TEOS : $0.13 \mathrm{FcC}_{16} \mathrm{Br}^{-} \mathrm{N}^{+}: 0.67 \mathrm{KCl}: 33.3 \mathrm{CH}_{3} \mathrm{CH}_{2} \mathrm{OH}$. Further, the reaction mixture was stirred at room temperature for $1 \mathrm{~h}$, and then transferred into an autoclave and left undisturbed at $180{ }^{\circ} \mathrm{C}$ for $12 \mathrm{~h}$. After hydrothermal treatment, the mixtures were evaporated and dried in a well ventilated space at room temperature. The samples were placed in a quartz boat and inserted into the quartz tube. Under a steady flow rate of $\mathrm{O}_{2}$, the temperature of the furnace was raised to $300{ }^{\circ} \mathrm{C}$ and maintained at this temperature for $6 \mathrm{~h}$. Subsequently, the furnace was slowly cooled down to room temperature, and then MMIOC were obtained. This sample was named as MMS.

2.2.3 Synthesis of $\mathrm{Fe}_{3} \mathrm{O}_{4}$. The sample was obtained through hydrothermal crystallization in alkaline conditions using a method reported in literature. ${ }^{27}$ The following reactants were used: ammonium ferrous sulfate as iron source and hydrazine hydrate as alkali source. The $\mathrm{pH}$ value of the reaction solution was adjusted to 11 using $0.5 \mathrm{M}$ sodium hydroxide solution. The resultant powder obtained was named as $\mathrm{Fe}_{3} \mathrm{O}_{4}$.

2.2.4 Synthesis of MMC with inorganic iron nitrate as iron precursor. MMC were synthesized in neutral medium through an evaporation induced self-assembly as previously reported. ${ }^{28}$ The following reactants were used: TEOS as the silica source, inorganic iron nitrate for iron resource, triblock copolymer P123 or F127 as template and 1,3,5-trimethylbenzene as cosolvent. The resultant powder obtained follows molar ratio of $\mathrm{Fe}\left[\mathrm{Fe}\left(\mathrm{NO}_{3}\right)_{3} \cdot 9 \mathrm{H}_{2} \mathrm{O}\right]: \mathrm{Si}$ as $8 / 2$ and $7 / 3$. Hence, the samples were named as $\mathrm{P} 8 / 2$ and $\mathrm{P} 7 / 3$ with $\mathrm{P} 123$, while they were named as F8/2 and F7/3 with F127.

2.2.5 Characterization. X-ray diffraction (XRD) patterns were obtained at room temperature on an X'Pert MPD pro diffractometer (Panalytical, the Netherlands) using Ni-filtered $\mathrm{Cu} \mathrm{K} \alpha$ radiation $(\lambda=1.5418 \mathrm{~nm})$ at $40 \mathrm{kV}$ and $10 \mathrm{~mA}$. Nitrogen adsorption and desorption isotherms were measured at $77 \mathrm{~K}$ using an AUTOSORB-1 analyzer (Contador's American). Surface areas were calculated by Brunauer-Emmett-Teller (BET) method. The pore size distribution was calculated from desorption branches of nitrogen isotherms using Barret-Joyner-Halenda (BJH) model. Scanning electron microscopy (SEM) images were acquired using a JEOL JSM-6700F field emission SEM $(20 \mathrm{kV})$. Transmission electron microscope (TEM) images were recorded on Tecnai F30 TEM (FEI, the Netherlands) at a working voltage of $300 \mathrm{kV}$. Magnetic performance was carried out using a lakeshore 7407 vibrating sample magnetometer (VSM, American) with fields up to 20000 Oe and at room temperature. X-ray photoelectron spectroscopy (XPS) measurements were performed on a ThermoFisher ESCALAB ${ }^{\mathrm{TM}}$ 250Xi System with $\mathrm{Al} \mathrm{K} \alpha$ source. All binding energies were referenced to the $\mathrm{C} 1 \mathrm{~s}$ peak at $284.6 \mathrm{eV}$ of surface adventitious carbon. Electron paramagnetic resonance (EPR spectrometer, JESFA200) was performed using 5,5-dimethyl-1-pyrrolidine- $N$ oxide (DMPO) as a spin-trapping agent. Metal content in the solution was determined by atomic absorption spectrometer (A8800) from American PE.

\subsection{Experimental procedures}

Catalytic degradation experiments were performed in a series of $250 \mathrm{~mL}$ conical flasks on a controlled temperature shaking table. Typically, $2 \mathrm{~g} \mathrm{~L}^{-1}$ catalytic agent was added into $100 \mathrm{~mL}$ $\mathrm{RhB}(1 \mathrm{mM})$ stock solution and shaken to achieve uniform mixing. Through pre-adsorption experiment, catalytic experiment was initiated following the introduction of $0.9128 \mathrm{~g}$ PS. The initial $\mathrm{pH}$ of the solution was adjusted using $2 \mathrm{M} \mathrm{HCl}$ or $\mathrm{NaOH}$. At a given time interval, a certain amount of sample was absorbed with methanol or TBA to quench primary radical species. The catalyst was separated from reaction fluid by 
centrifugation. The concentration of RhB was analyzed using TU-1901 automatic scanning UV-Vis spectrophotometer (Shanghai Analytical instruments Co. Ltd.) with a spectrometric quartz cell $(1 \mathrm{~cm})$. The value of COD was determined using spectrophotometric method. Degradation mechanism of RhB was analysed using an Agilent 7890A-5975 GC-MS analyzer. TOC data was acquired from a SHIMADZU TOC-V-CPH analyzer. All test performances were repeated at least three times to ensure smoothing error and correction data accuracy. A plot of $C v s$. $t$ was obtained, where $C_{0}$ was the initial concentration of RhB and $C$ was the concentration at time $t$; decolorization efficiency was obtained based on $\left(C_{0}-C\right) / C_{0}$.

\section{Results and discussion}

\subsection{Fabrication and characterization of MMIOC}

The overall structure was designed as follows: given surfactant containing ferrocene as both template agent and iron precursors was used to devise desired MMIOC. During the formation of MMIOC, the hydrophobic ferrocene tails were closely packed in the center of the micelles, which were used as structuredirecting agents for mesoporous channels. The iron resources were oxidized when ferrocene surfactant was removed by calcination in $\mathrm{O}_{2}$ atmosphere. Following this, highly dispersed iron ions were embedded in the channels. Clearly, this one-step self-assembly and oxidation process provided a simple and convenient strategy for preparing MMIOC.

As shown in Fig. 1(a), the addition of ferrocene surfactant as template agent provokes one strong and two very weak diffraction peaks, which indicates that ferrocene terminal groups could penetrate into the hydrophobic core of surfactant micelle. Such a ferrocene terminal group leads to the micelle deformation into sphericity, which eventually assembles to form porous materials. The absence of diffraction peak would be ascribed to its non-porous structure. ${ }^{5}$ However, by low-angle XRD measurements, the diffractogram might be attributed to honeycomb mesoporous structure. Wide-angle X-ray diffraction (XRD) analyses were performed to corroborate the presence of metal oxides deposited on the support and its poor crystallinity. As shown in Fig. 1(b), diffraction signals at $2 \theta$ equal to 33.2, $35.7,49.6,54.2$ and $62.6^{\circ}$ could be assigned to magnetic $\alpha-\mathrm{Fe}_{2} \mathrm{O}_{3}$ crystallites (JCPDS \#84-0306). ${ }^{29}$

Transmission electron micrographs for MMS are shown in Fig. 2. Clearly, magnetic iron oxide was embedded in the mesoporous channel (Fig. 2(b)). Honeycomb mesoporous structure with pore diameter of about $2.0 \mathrm{~nm}$ displays local and less longrange order, in accordance with small-angle XRD patterns (Fig. 2(a)). ${ }^{30}$ Field dependence hysteresis loop for MMS at $293 \mathrm{~K}$ is shown in Fig. 3S. $\dagger$ The measured coercive force, $H_{\mathrm{c}}$, was 316.23 Oe, the magnetization saturation value $M_{\mathrm{s}}$ was 0.8046 emu $\mathrm{g}^{-1}$ and the remanence was $0.2666 \mathrm{emu} \mathrm{g}^{-1}$. Hysteresis behavior is a consequence of surface spin disorder, showing soft ferromagnetic characteristics that are different from the reported antiferromagnetic characteristics. ${ }^{31}$

Further, the mesoporous structure was confirmed by nitrogen adsorption-desorption. It could be observed that MMS displayed Langmuir type IV isotherms (Fig. 3(a)), corresponding to typical mesoporous materials. The isotherms were clearly different from those of $\mathrm{H} 1$ hysteresis loop. Notably, there is still a large step observed at a relative pressure of $0.4-0.95$, which is
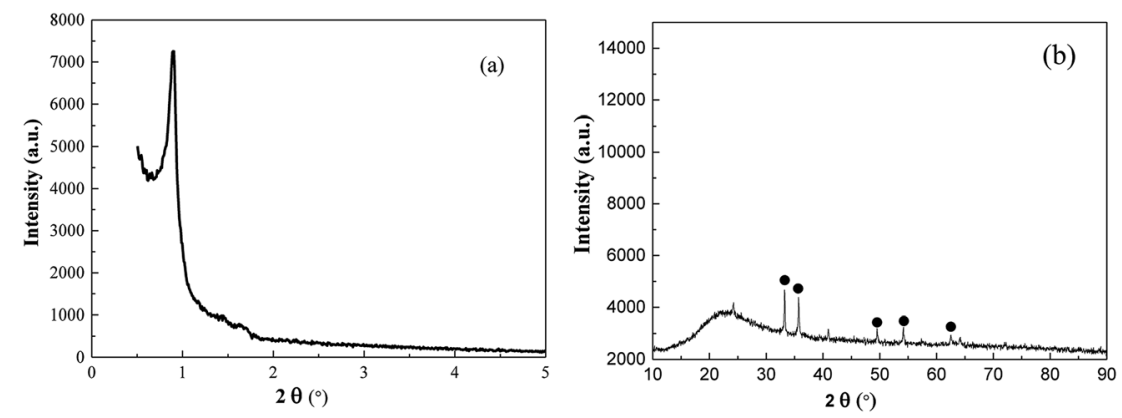

Fig. 1 Small-angle (a) and wide-angle (b) XRD patterns for MMS.
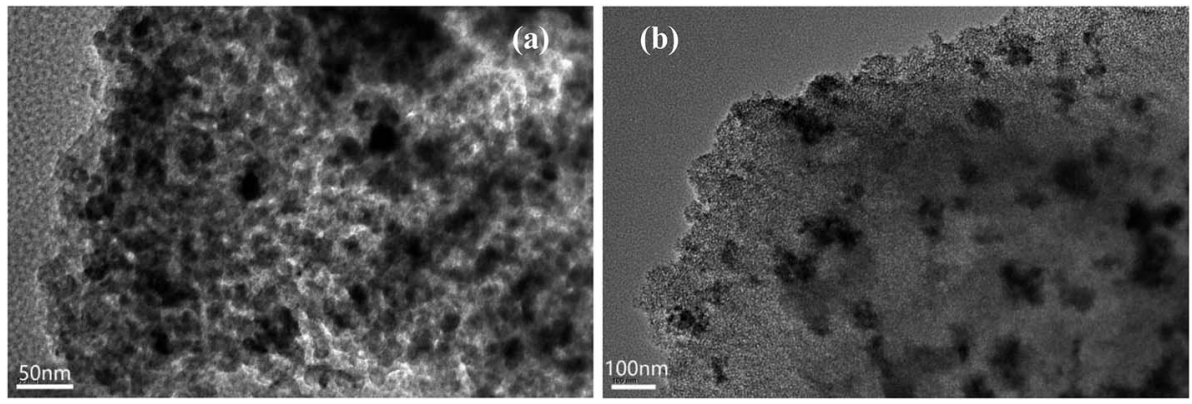

Fig. 2 TEM images of MMIOC for MMS. 

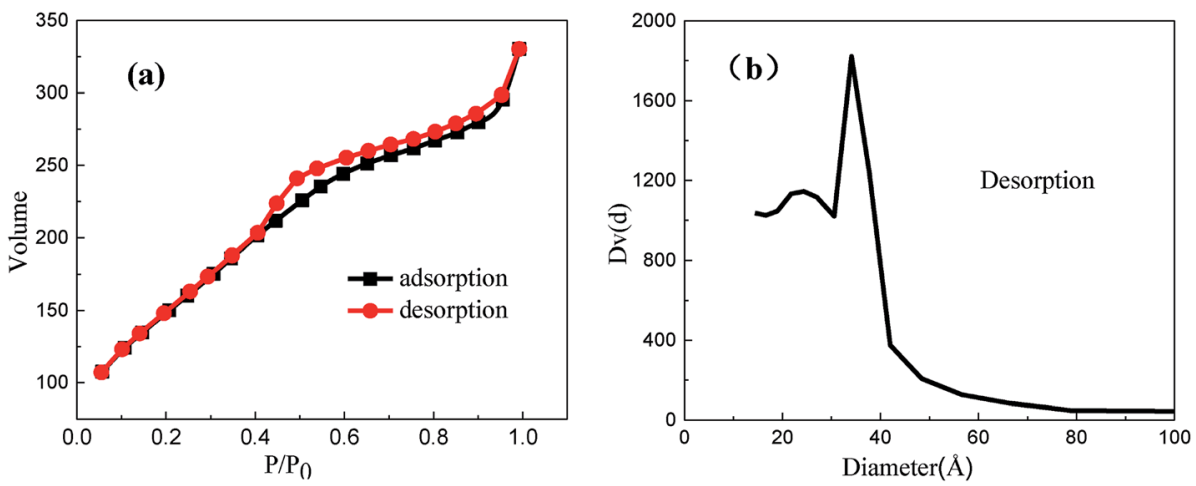

Fig. 3 Nitrogen sorption isotherms (a) and pore size distribution (b) obtained from analysis of desorption branch of isotherms for the MMS.

in agreement with characteristic results of TEM images, indicating the presence of honeycomb mesopores. ${ }^{32}$ However, type H1 hysteresis has been usually observed on solids with cylindrical mesopores. ${ }^{33}$ MMS have relatively high surface area $\left(550.1 \mathrm{~m}^{2} \mathrm{~g}^{-1}\right)$ in contrast to the composite reported in previous literature. ${ }^{5}$ Furthermore, the total pore volume was $0.5077 \mathrm{~cm}^{3} \mathrm{~g}^{-1}$. The mesoporous size distribution exhibits a sharp peak centered at about $2.4 \mathrm{~nm}$ (Fig. 3(b)).

The MMS morphology showed no fixed shapes (Fig. 4(a)). Fig. $4(\mathrm{~b}-\mathrm{d})$ shows $\mathrm{Fe}, \mathrm{Si}$ and $\mathrm{O}$ elements surface distribution, respectively. The EDS results shows that the atomic percentage of silicon, oxygen and iron are $16.9 \%, 79.9 \%$ and $3.2 \%$, respectively (Fig. 5S $\dagger$ ). The Fe elements map showed that iron oxides were highly dispersed in mesoporous framework in order to increase its specific surface area and improve its catalytic activity. Si and O elements were important components of the mesoporous framework.
$\mathrm{X}$-ray photoelectron spectroscopy (XPS) is one of the most powerful tools to investigate surface element composition and their valence states; the XPS spectra of MMS and $\mathrm{Fe}_{3} \mathrm{O}_{4}$ samples are shown in Fig. 5. The full XPS spectrum clearly showed the presence of $\mathrm{C}, \mathrm{Si}, \mathrm{O}$ and $\mathrm{Fe}$ element on MMS sample surface, while only $\mathrm{C}, \mathrm{O}$ and $\mathrm{Fe}$ elements were found on $\mathrm{Fe}_{3} \mathrm{O}_{4}$ sample surface (Fig. 5(A)). As shown in Fig. 5(B), XPS O (1s) of $\mathrm{Fe}_{3} \mathrm{O}_{4}$ sample envelope was deconvoluted into three parts, namely, lattice oxygen at $530.5 \mathrm{eV}$, hydroxylate oxygen at $531.8 \mathrm{eV}$ and the oxygen adsorbed on $\mathrm{H}_{2} \mathrm{O}$ at $533.0 \mathrm{eV} ;^{34}$ for the spectrum $\mathrm{O}$ (1s) of the MMS, four single peaks with binding energies of 530.4, 531.4, 532.7 and $533.0 \mathrm{eV}$ corresponded to lattice oxygen, hydroxylate oxygen, silica oxide and adsorbed water, respectively. ${ }^{35}$ Clearly, silica frameworks have been verified for MMS samples. Core-level Fe 2p spectra have evidently shown two kinds of binding energies of 711.36 and $724.68 \mathrm{eV}$ ascribed to the $\mathrm{Fe} 2 \mathrm{p}_{3 / 2}$ and $\mathrm{Fe} 2 \mathrm{p}_{1 / 2}$, respectively, and the binding energy of $719.37 \mathrm{eV}$ was ascribed to the satellite peak (denoted as Sat.)
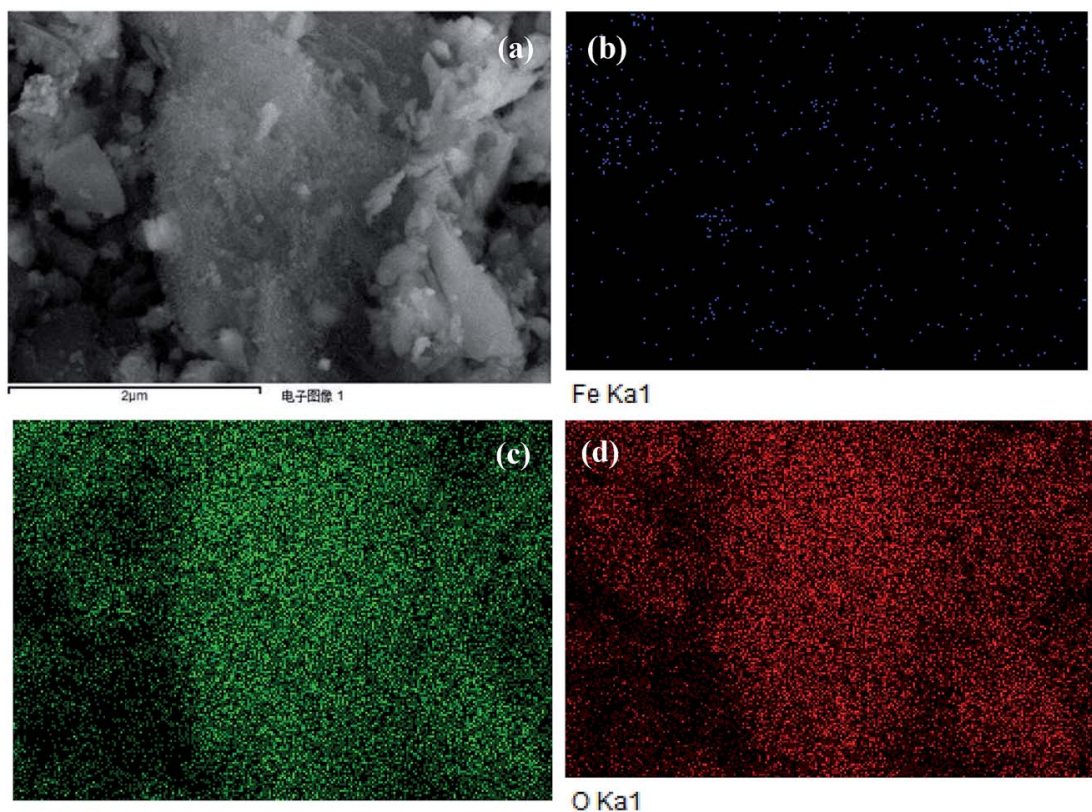

Fe Ka1

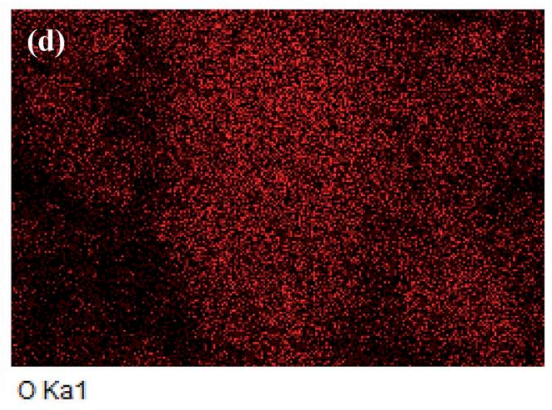

Si Ka1

Fig. 4 SEM photographs (a) element (b) Fe, (c) Si and (d) O mapping for MMS. 

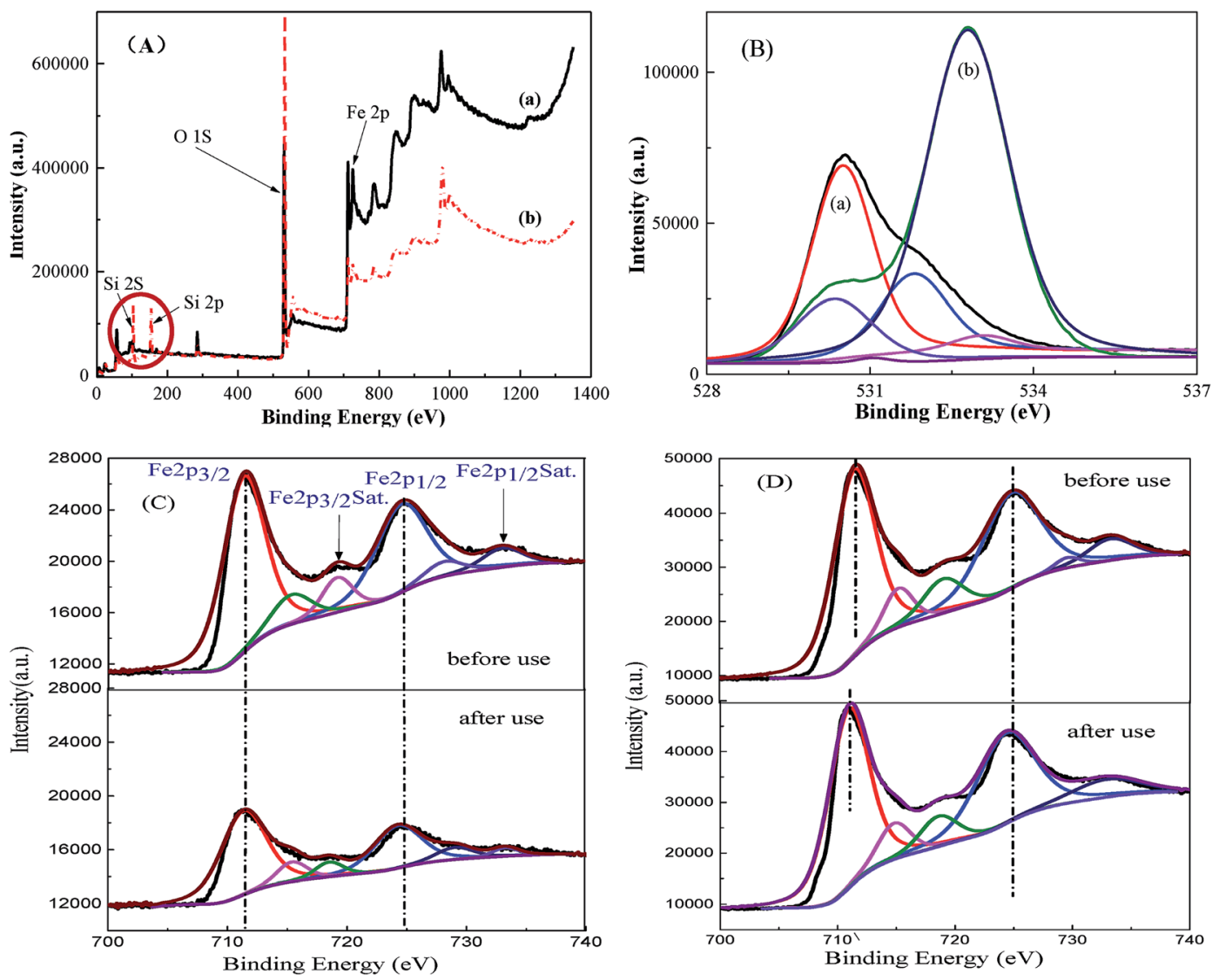

Fig. 5 Full spectra (A) and $\mathrm{O}$ 1s XPS spectra (B) for (a) $\mathrm{Fe}_{3} \mathrm{O}_{4}$ and (b) MMS; Fe $2 \mathrm{p}$ XPS spectra for (C) $\mathrm{MMS}$ and (D) $\mathrm{Fe}_{3} \mathrm{O}_{4}$.

(Fig. 5(C)). ${ }^{36-38}$ The peak intensities of $\mathrm{Fe} 2 \mathrm{p}_{3 / 2}$ and $\mathrm{Fe} 2 \mathrm{p}_{1 / 2}$, after repeated use for 10 times, was weaker than that obtained before use, accompanied by broadening of their peak shapes. This suggested leakage of iron ions on the surface of the samples. ${ }^{36}$ However, peak intensity and broadening of $\mathrm{Fe} 2 \mathrm{p}_{3 / 2}$ and $2 \mathrm{p}_{1 / 2}$, and their satellite peaks have scarcely changed in case of $\mathrm{Fe}_{3} \mathrm{O}_{4}$ (Fig. 5(D)). This means that there was no change in surface element composition before and after use. Furthermore, the leakage of iron ions leads to a reduction of magnetic iron oxide blocks. In summary, the leakage of iron ions on the surface of the samples before and after use was confirmed.

Fe $2 \mathrm{p}_{3 / 2}$ spectra was divided into a strong peak $(711.25 \mathrm{eV})$ and a small shoulder (710 eV) attributed to $\mathrm{Fe}(\mathrm{III})$ and $\mathrm{Fe}(\mathrm{II})$, respectively (Fig. $6 \mathrm{~S}(\mathrm{~A}) \dagger) .{ }^{39,40}$ The satellite signal $(719.37 \mathrm{eV})$ suggested the presence of ferrous oxide. It was found that $\mathrm{Fe}(\mathrm{III})$ : $\mathrm{Fe}(\mathrm{II})$ on the catalyst surface was 5.7 (Table $1 \mathrm{~S} \dagger$ ). Owing to charge effect, the entire binding energy spectra shifted before and after use for the MMS and $\mathrm{Fe}_{3} \mathrm{O}_{4} \cdot{ }^{41,42}$ This may be ascribed
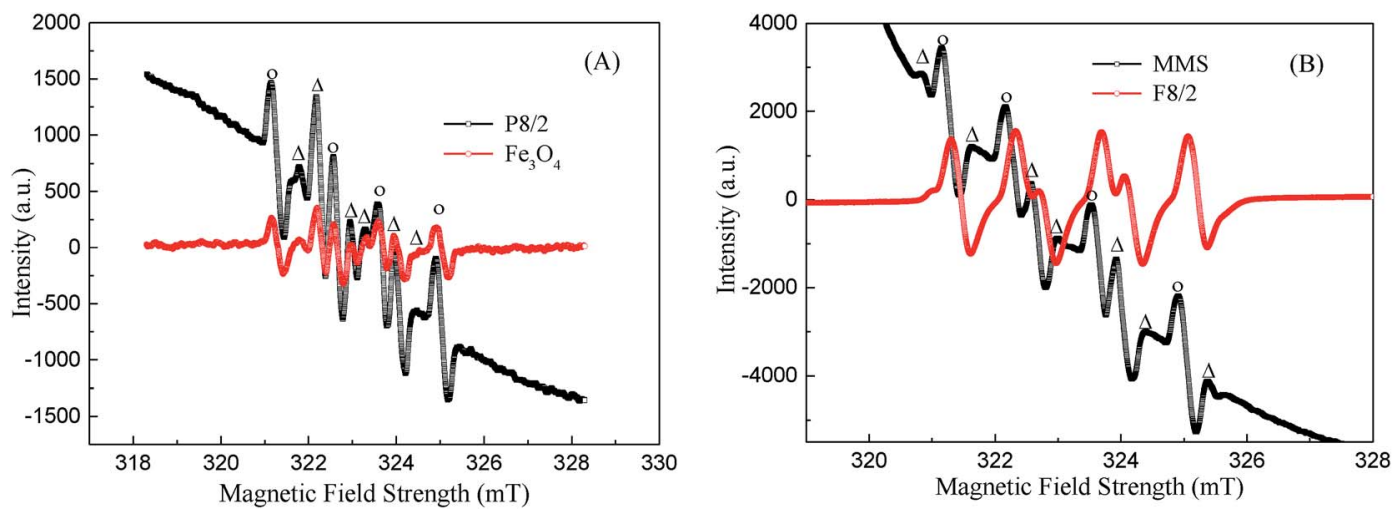

Fig. 6 EPR spectra obtained through MMIOC systems: (A) P8/2 and $\mathrm{Fe}_{3} \mathrm{O}_{4}$; (B) MMS and F8/2; activated PS oxidative processes in the presence of DMPO. [PS] $=40 \mathrm{mM}$; $\left[\mathrm{MMIOC}\right.$ or $\left.\mathrm{Fe}_{3} \mathrm{O}_{4}\right]=5 \mathrm{~g} \mathrm{~L}^{-1}$; $[\mathrm{DMPO}] \approx 0.1 \mathrm{M} ; \mathrm{pH}=7.0 \pm 0.5 ; T=25^{\circ} \mathrm{C}$. 

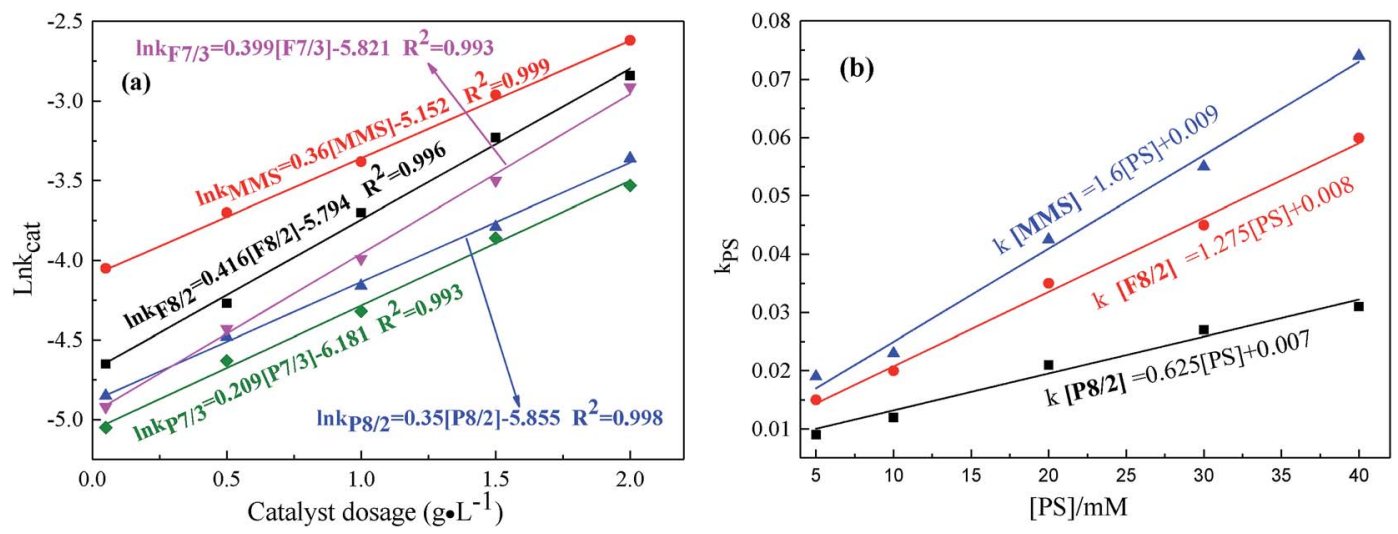

Fig. 7 Effects of (a) catalyst dosage; (b) PS concentration on RhB degradation in different $\mathrm{Fe}_{x} \mathrm{O}_{y} / \mathrm{PS}$ systems. Expect investigated parameter, others fixed at $[\mathrm{RhB}]=1 \mathrm{mM}$; initial $\mathrm{pH}=7.0 ; T=25^{\circ} \mathrm{C}$.

to iron ions partially dissolved during the degradation of RhB. However, these lattice positively-charged Fe groups could function as active sites for PS decomposition to generate the radicals and degrade the pollutants.

To elucidate the mechanism of PS activation on MMIOC systems of RhB degradation, in situ electron paramagnetic resonance (EPR) were performed, in which DMPO was used as spin trapping agent for $\mathrm{SO}_{4}{ }^{--}$and ${ }^{\circ} \mathrm{OH}$ radicals. Fig. 6 shows that the signals of both $\mathrm{DMPO}^{-\mathrm{SO}_{4}}{ }^{-{ }^{-}}$and DMPO- ${ }^{-} \mathrm{OH}$ have been easily detected in MMIOC/PS systems, meaning that the obtained MMIOC systems are effective for RhB degradation.

The results obtained shows that MMS had excellent ability for catalytic activation of PS to generate active radicals. It has been demonstrated that PS could be activated by transition metal ions $\mathrm{Fe}^{2+}$ to generate sulfate radical $\left(\mathrm{SO}_{4}{ }^{-{ }^{-}}\right)\left(E_{0}=2.6\right.$ $\mathrm{eV})$. Furthermore, hydroxyl radical $\left({ }^{\circ} \mathrm{OH}^{-}\right)\left(E_{0}=2.8 \mathrm{eV}\right) . \equiv \mathrm{Fe}^{3+}$ was reduced to $\equiv \mathrm{Fe}^{2+}$, to produce PS radical, as electron donor, on MMIOC surface (eqn (1)). ${ }^{43}$ However, the intensity of DMPO-OH signals was much stronger than that of $\mathrm{DMPO}^{-\mathrm{SO}_{4}}$ due to fast transformation from $\mathrm{DMPO}-\mathrm{SO}_{4}$ to DMPO-OH via nucleophilic substitution (eqn (3) and (4)) ${ }^{44}$ These were the key steps in reaction system. This system could be applied in the degradation of $\mathrm{RhB}$, as typical representative of dye wastewater (eqn (5)).

$$
\begin{gathered}
\equiv \mathrm{Fe}(\mathrm{III})+\mathrm{S}_{2} \mathrm{O}_{8}{ }^{2-} \rightarrow \equiv \mathrm{Fe}(\mathrm{II})+\mathrm{S}_{2} \mathrm{O}_{8}{ }^{\cdot-} \\
\equiv \mathrm{Fe}(\mathrm{II})+\mathrm{S}_{2} \mathrm{O}_{8}{ }^{2-} \rightarrow \equiv \mathrm{Fe}(\mathrm{III})+\mathrm{SO}_{4}{ }^{\cdot-}+\mathrm{SO}_{4}{ }^{2-} \\
\mathrm{SO}_{4}{ }^{-{ }^{-}}+\mathrm{H}_{2} \mathrm{O} \rightarrow \cdot \mathrm{OH}+\mathrm{H}^{+}+\mathrm{SO}_{4}{ }^{2-} \\
\mathrm{SO}_{4}{ }^{--}+\mathrm{HO}^{-} \rightarrow \cdot \mathrm{OH}+\mathrm{SO}_{4}{ }^{2-} \\
\mathrm{RhB}+\mathrm{SO}_{4}{ }^{\cdot-} / \cdot \mathrm{OH} \rightarrow \mathrm{CO}_{2}+\mathrm{H}_{2} \mathrm{O}+\mathrm{N}_{2}
\end{gathered}
$$

As the iron component possesses a single electron in the $3 \mathrm{~d}$ orbital, MMIOC could serve as a medium with high specific surface area and highly dispersed catalytic activity, to facilitate electron transfer from the iron to the PS to produce ${ }^{\circ} \mathrm{OH}$ and $\mathrm{SO}_{4}{ }^{--}$radicals. In contrast, bulk magnetic iron oxides presents relatively low catalytic effectiveness for PS activation, ${ }^{45}$ which could be due to partial electrons confined in crystalline structure. Thus, based on below-mentioned experimental results and detailed discussion, possible mechanism of PS activation on MMIOC systems of RhB degradation has been depicted in Scheme 1.

\subsection{Degradation performance of MMIOC}

As shown in Fig. 7(a and b), the influences of catalyst dosage and PS concentration on RhB degradation were confirmed in

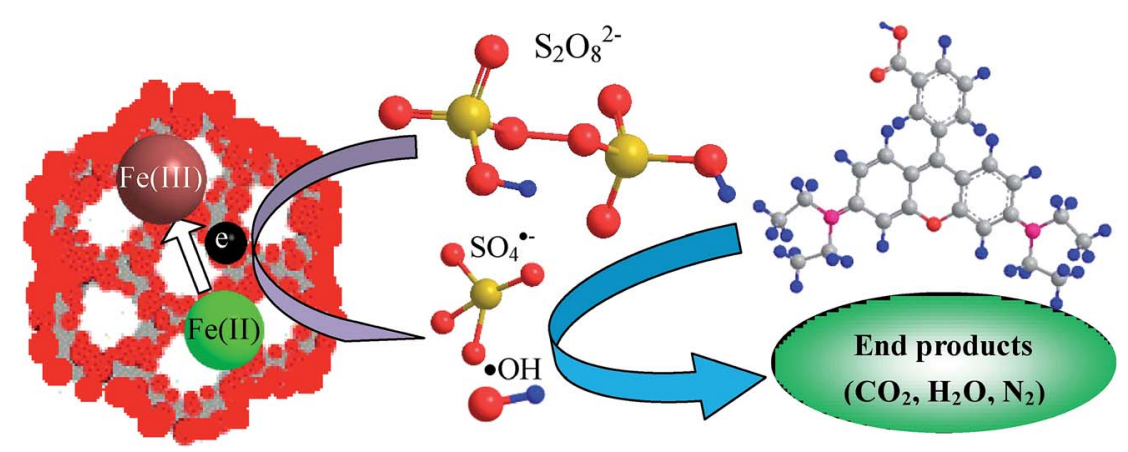

Scheme 1 Activation mechanism of PS by MMS. 

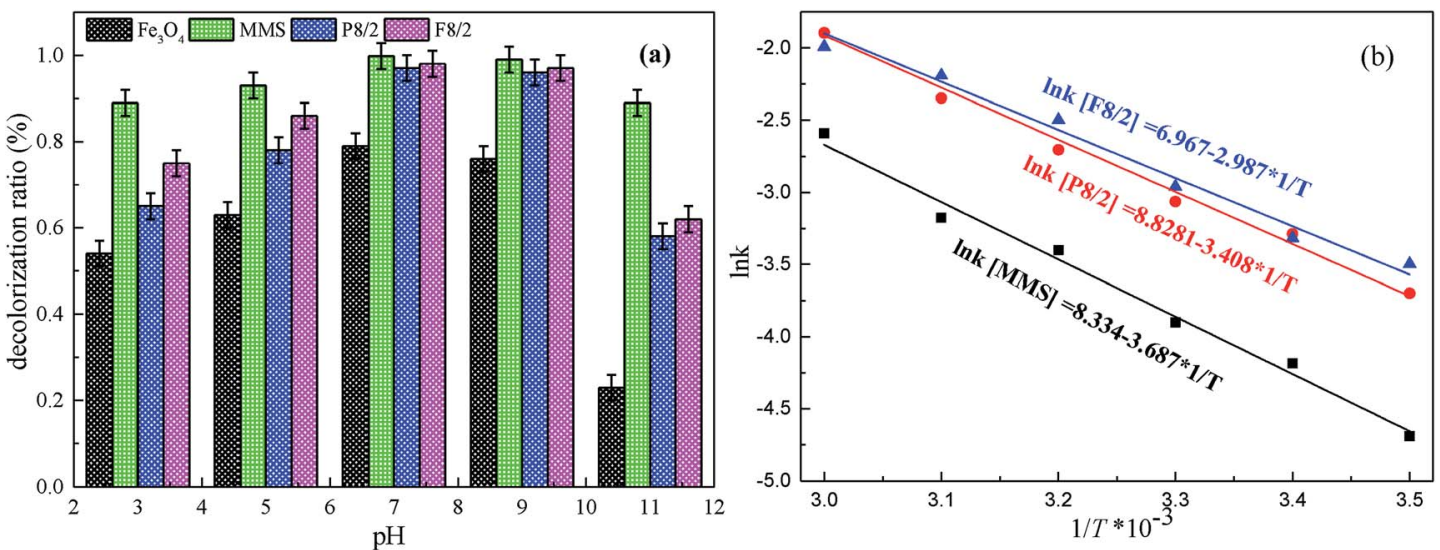

Fig. 8 Effects of (a) $\mathrm{pH}$, (b) temperature on $\mathrm{RhB}$ degradation in different $\mathrm{Fe}_{x} \mathrm{O}_{y} / \mathrm{PS}$ systems. Expect investigated parameter, others fixed at $[\mathrm{RhB}]=$ $1 \mathrm{mM}$; [catalyst] $=2.0 \mathrm{~g} \mathrm{~L}^{-1}$; [PS] $=40 \mathrm{mM}$.

different MMIOC/PS systems. As shown, the degradation rate constant is pseudo-first-order with respect to catalyst dosage concentration. ${ }^{\mathbf{3 4}, 46}$ Their correlation coefficients were higher than $99 \%$. With the increase of the catalyst dosage from 0.05 to $2 \mathrm{~g} \mathrm{~L} \mathrm{~L}^{-1}$, degradation effect was improved significantly. The apparent enhancement could be accounted for large number of surface active sites to activate PS and rapidly form active radicals on higher catalyst dosage. ${ }^{47}$ The ratio of natural logarithm of degradation rate constant and catalyst dosage was significantly different in different MMIOC/PS systems. This could be attributed to the difference between mesoporous structure, surface iron content and catalyst dosage. Further, it is concluded that the degradation rate constant of MMS catalyst dosage was highest. Superior catalytic performance could be ascribed to magnetic component embedded in inner or outer surface of magnetic mesoporous materials, forming catalytic active sites, thus providing a new strategy to modulate their electronic structure, resulting in generating more radicals for RhB degradation. ${ }^{48}$ Degradation rate constant of $\mathrm{F}$ type catalyst is higher than that of $\mathrm{P}$ type. Except iron content of samples, pore structure of $\mathrm{F}$ type is more favorable for molecular transport relative to $\mathrm{P}$ type. Moreover, mesoporous structure of MMS is most favorable to improve degradation efficiency. Pore structure of $\mathrm{F}$ type might be inferred to the formation of a cagelike mesopore, ${ }^{49-55} \mathrm{P}$ type six square. ${ }^{56-59}$

Similar to the behavior of catalyst dosage, degradation rate constants also linearly increase as PS concentration increases from 5 to $40 \mathrm{mM}$ (Fig. 7(b)). ${ }^{34}$ In addition, degradation effect also improved significantly. With the increase of PS dosage, degradation ratio constant of MMS sample was the highest. At same PS dosage, the degradation ratio constant of F8/2 sample was higher than that of $\mathrm{P} 8 / 2$ sample. From a series of data it is inferred that, first, the pore structure corresponds to mass transportation, resulting in catalytic performance difference. Second, the specific surface area highly affects the degradation ratio constant.

The influence of initial $\mathrm{pH}$ from 3-11 on $\mathrm{RhB}$ degradation in different MMIOC/PS systems is displayed in Fig. 8(a). As observed, the best $\mathrm{RhB}$ degradation ratio occurs at $\mathrm{pH}$ 7-9, under or above which removal efficiency of $\mathrm{RhB}$ dramatically decreases. This revealed that PS could be effectively activated on as-prepared MMIOC surface and produce a large number of radicals to degrade $\mathrm{RhB}$ under neutral-alkaline conditions. Lower or higher $\mathrm{pH}$ value leads to lower degradation ratios. At higher $\mathrm{pH}$, the lower degradation ratio is due to charge repulsion in active site of mesoporous surface. Nevertheless, the iron species deactivates PS at lower $\mathrm{pH},{ }^{60}$ resulting in the decrease of degradation ratio. ${ }^{34}$

Fig. 8(b) presents the effect of reaction temperature on degradation ratios in various MMIOC/PS systems. As shown, the increase of reaction temperature accelerates the degradation ratio of the $\mathrm{RhB}$, which could be caused by rapid breakdown of PS into ${ }^{\circ} \mathrm{OH}$ or $\mathrm{SO}_{4}{ }^{\cdot-}$ under thermal activation..$^{9-11,61}$ Moreover, the systems could cross reaction energy barrier easily at higher temperature. $^{62}$ Furthermore, Arrhenius rate constants for various MMIOC/PS systems presented a good linear correlation with temperature. Calculated activation energy $\left(E_{\mathrm{a}}\right)$ values were

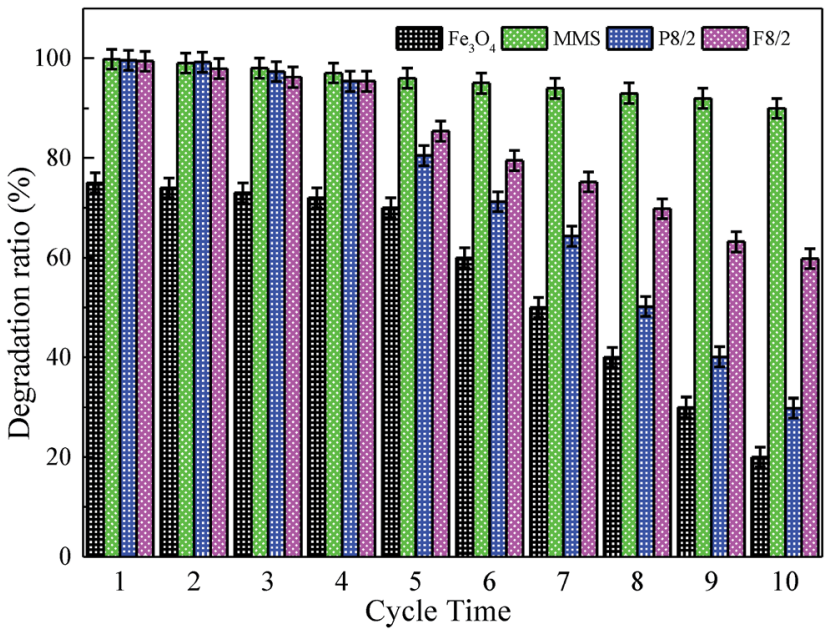

Fig. 9 Effect of reusable times on $\mathrm{RhB}$ degradation in different $\mathrm{Fe}_{x} \mathrm{O}_{y} /$ PS systems. Expect investigated parameter, others fixed at $[\mathrm{RhB}]=$ $1 \mathrm{mM}$; [catalyst $]=2.0 \mathrm{~g} \mathrm{~L}^{-1}$; [PS] $=40 \mathrm{mM}$; initial $\mathrm{pH}=7.0 ; T=25^{\circ} \mathrm{C}$. 


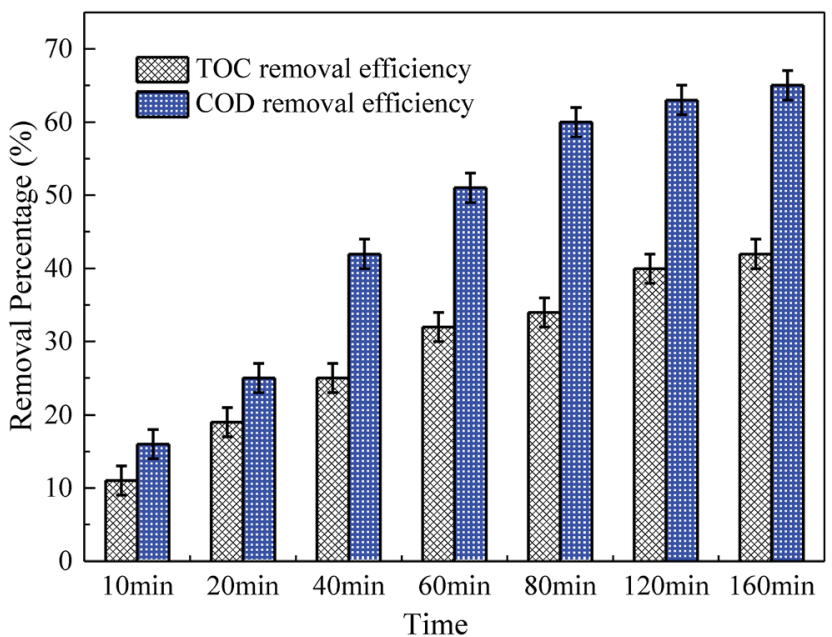

Fig. 10 The variation of TOC and COD with reaction time on RhB degradation in MMS/PS systems. Expect investigated parameter, others fixed at $[\mathrm{RhB}]=1 \mathrm{mM}$; [catalyst $]=2.0 \mathrm{~g} \mathrm{~L}^{-1}$; $[\mathrm{PS}]=40 \mathrm{mM}$; initial $\mathrm{pH}=7.0 ; \mathrm{T}=25^{\circ} \mathrm{C}$

30.65, 28.33 and $24.84 \mathrm{~kJ} \mathrm{~mol}^{-1}$ for $\mathrm{P} 8 / 2, \mathrm{~F} 8 / 2$ and MMS systems, respectively. Cai et $a l .{ }^{63}$ reported a US/Fe-Co/SBA-15/ PMS system for the degradation of orange II and measured the activation energy to be about $10.5 \mathrm{~kJ} \mathrm{~mol}^{-1}$. Activation energies of $\mathrm{Co}_{3} \mathrm{O}_{4}$ catalyst systems for phenol degradation are in the range of $47-70 \mathrm{~kJ} \mathrm{~mol}^{-1} \mathbf{. 6 4}^{\mathbf{4}}$

The RhB degradation performance in aqueous solution was estimated by various $\mathrm{Fe}_{x} \mathrm{O}_{y} / \mathrm{PS}$ systems under optimum conditions. As shown in Fig. 7S and 8S, $\dagger$ complete degradation of $1 \mathrm{mM}$ RhB was obtained within 120 minutes on various MMIOC/PS systems. Degradation performance could be basically fitted by first-order kinetic model..$^{65,66}$ The RhB degradation ratio was below $80 \%$ on block $\mathrm{Fe}_{3} \mathrm{O}_{4} / \mathrm{PS}$ system in the absence of mesoporous structure, implying to eliminate the influence of diffusive mass transfer and adsorption. ${ }^{66}$
Recently, related papers have reported iron loading advanced oxidation process. ${ }^{\mathbf{6 3 , 6 7 , 6 8}}$ The key problem was to solve iron leakage, catalyst deactivation, which caused additional contamination. Fig. 9 presents the stability and repetitive use rate of the catalyst by cyclic reuse on various $\mathrm{Fe}_{x} \mathrm{O}_{y} / \mathrm{PS}$ systems. In each test, the catalyst was separated by centrifugation; then, it was washed with an excess amount of deionized water and dried. The XPS spectra displayed chemical component change on catalyst surface before and after 10 times of repeated usage. The recycled catalyst maintained its original iron red color. Approximate $90 \%$ of degradation ratio was obtained for ten times of recycle on MMS/PS systems. MMS/PS systems showed a good regeneration and reusability, and so did the Fe/OMC system. ${ }^{69}$ Furthermore, MMS/PS system degraded organic pollutants by oxidation, while Fe/OMC system removed organic pollutants by adsorption. Therefore, Fe/OMC system required additional desorption steps to regenerate. For block $\mathrm{Fe}_{3} \mathrm{O}_{4} / \mathrm{PS}$ system, only $70 \%$ of the decolorization efficiency was obtained in the first five cycles. With the increase of cycles the degradation ratio decreased gradually, and for tenth cycle only $20 \%$ of degradation efficiency was observed. Small specific surface area of magnetic blocks and an iron leakage for the $\mathrm{Fe}_{3} \mathrm{O}_{4} / \mathrm{PS}$ system led to largely ineffective activation of PS. However, superior reusability of MMS could be ascribed to magnetic iron species inlayed or embedded in inner or outer surface of magnetic mesoporous materials, forming catalytic active site. The iron content of MMS catalysts was measured by EDS and Fe elements map. After removing the catalyst by centrifugation of MMS/PS system, iron content in centrifugal solution was determined by atomic adsorption. The catalyst was reused several times, and each time the iron content in centrifugal solution was less than 1 ppm.

The variation of TOC and COD were determined to clarify the mineralization in MMS/PS systems. As shown in Fig. 10, the removal efficiencies of TOC and COD after $60 \mathrm{~min}$ reaction were $32 \%$ and $51 \%$, respectively. This could be explained by the fact

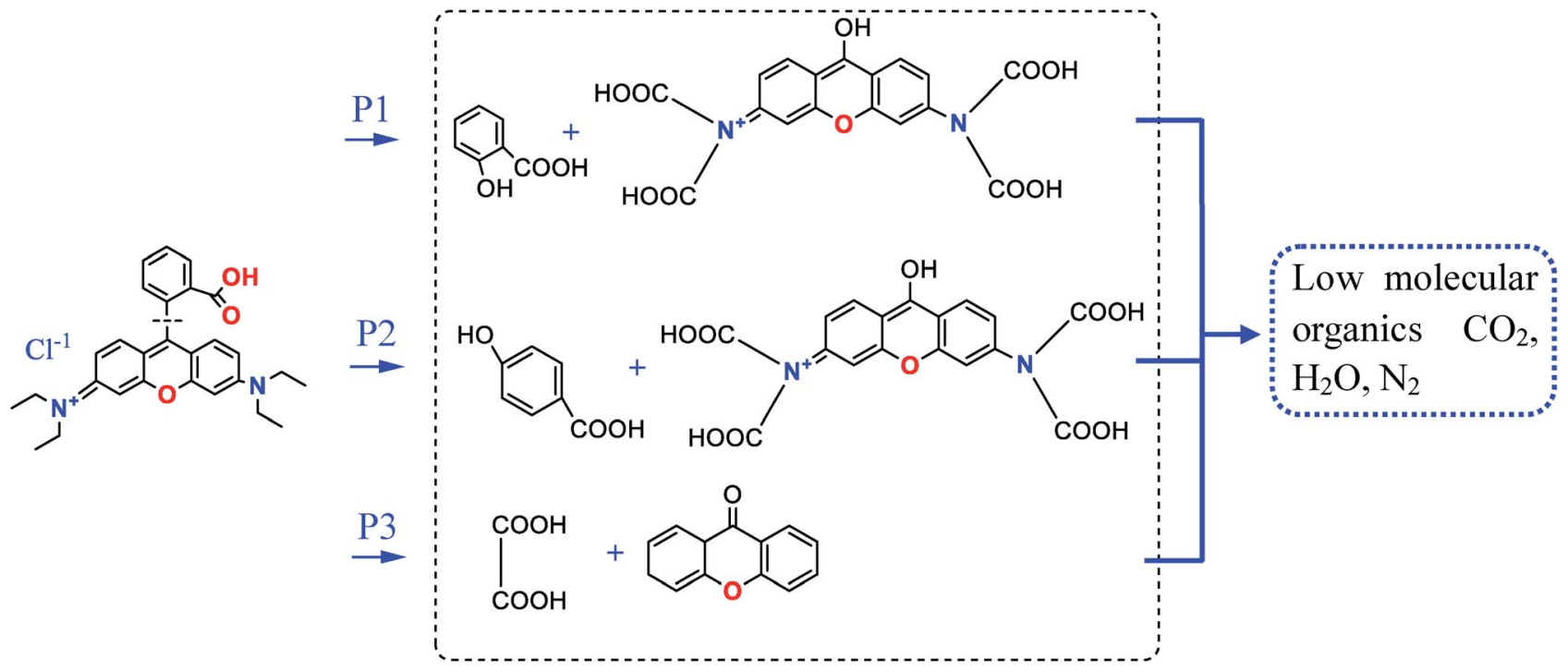

Fig. 11 Degradation pathways of RhB in MMS/PS system. 
that the RhB was difficult to completely decolorize and the oxidation rate of intermediates could be much slower than RhB. ${ }^{63}$ Therefore, relatively more time was required to obtain a favorable mineralization, compared with RhB decolorization. When the reaction time was extended to $160 \mathrm{~min}$, the removal efficiencies of TOC and COD increased to $42 \%$ and $65 \%$, respectively. This showed the increase in the mineralization performance of the MMS/PS systems.

On the MMS/PS systems, RhB degradation was divided into several stages. The molecular type at each stage of degradation was identified by an Agilent 7890A-5975 GC-MS analyzer. It has been reported previously that the introduction of ordered mesoporous graphitic carbon nitride could successfully and rapidly decolorize $\mathrm{RhB} .{ }^{70}$ On the basis of free radicals formation, oxidative decarbonization and RhB degradation efficiency, this study generally analysed the mechanism of RhB degradation. Oxidation and rearrangement of the molecular structure on the MMS/PS systems are shown in Fig. 11. According to these organic intermediates, RhB degradation scheme containing three possible transformation pathways could be proposed. With respect to three degradation pathways initiated by ${ }^{\circ} \mathrm{OH}$ or $\mathrm{SO}_{4}{ }^{-}$, these unstable intermediates would open the rings and form low molecular organics, leading to mineralized inorganic compounds. The above result also evidenced that ${ }^{\circ} \mathrm{OH}$ and $\mathrm{SO}_{4}{ }^{\cdot-}$, which were generated by mesoporous embedded $\equiv \mathrm{Fe}^{3+}$ activated PS could chiefly participate in degradation of RhB in MMS/PS system.

\section{Conclusions}

By employing hydrophobic ferrocene surface active agent as the micelles, MMIOC were successfully prepared via evaporation induced self-assembly and hydrothermal method. Iron ions were embedded in inner or outer surface, forming catalytic active sites that provide new strategy to modulate their electronic structure, resulting in generating radicals for efficient degradation of RhB. There is a linear relationship between various parameters, such as catalyst dose, PS dose, systematic temperature and degradation ratio constant. Under optimum conditions, degradation efficiency reached 99\% after $60 \mathrm{~min}$ reaction, and increased to $99.8 \%$ when the reaction time was extended to $120 \mathrm{~min}$. Degradation efficiency was compared between MMIOC/PS and $\mathrm{Fe}_{3} \mathrm{O}_{4} / \mathrm{PS}$ systems. Mesoporous structure and magnetism could synergistically improve degradation performance and increase the stability and reusability of the catalyst. Therefore, heterogeneous MMIOC/PS activation systems could be a potential technology for organic wastewater treatment.

\section{Conflicts of interest}

There are no conflicts to declare.

\section{Acknowledgements}

The study was financially supported by the National Young Natural Science Foundation of China (No. 21307169). The author wishes to thank the National Nature Science Foundation of China for their financial support.

\section{References}

1 A. Uygur and E. Kök, Color. Technol., 1999, 115, 350.

2 S. Larouk, R. Ouargli, D. Shahidi, L. Olhund, T. C. Shiao, N. Chergui, T. Sehili, R. Roy and A. Azzouz, Chemosphere, 2017, 168, 1648.

3 R. F. Dantas, S. Contreras, C. Sans and S. Esplugas, J. Hazard. Mater., 2008, 150, 790.

4 A. G. Trovó, R. F. P. Nogueira, A. Agüera, C. Sirtori and A. R. Fernández-Alba, Chemosphere, 2009, 77, 1292.

5 J. Deng, S. F. Feng, K. J. Zhang, J. Li, H. Y. Wang, T. Q. Zhang and X. Y. Ma, Chem. Eng. J., 2017, 308, 505.

6 G. P. Anipsitakis and D. D. Dionysiou, Environ. Sci. Technol., 2004, 38, 3705.

7 J. Deng, Y. S. Shao, N. Y. Gao, C. Q. Tan, S. Q. Zhou and X. H. Hu, J. Hazard. Mater., 2013, 262, 836.

8 C. Gong, F. Chen, Q. Yang, K. Luo, F. B. Yao, S. N. Wang, X. L. Wang, J. W. Wu, X. M. Li, D. B. Wang and G. M. Zeng, Chem. Eng. J., 2017, 321, 222.

9 A. Ghauch, A. M. Tuqan and N. Kibbi, Chem. Eng. J., 2012, $197,483$.

10 A. Ghauch and A. M. Tuqan, Chem. Eng. J., 2012, 183, 162.

11 A. Ghauch, A. M. Tuqan and N. Kibbi, Chem. Eng. J., 2015, 279, 861.

12 A. Ghauch, A. Baalbaki, M. Amasha, R. E. Asmar and O. Tantawi, Chem. Eng. J., 2017, 317, 1012.

13 Z. F. Ma, Y. Yang, Y. H. Jiang, B. D. Xi, T. X. Yang, X. Peng, X. Y. Lian, K. Yan and H. L. Liu, Chem. Eng. J., 2017, 311, 183.

14 Y. Li, Y. Li, S. Ma, P. Wang, Q. Hou, J. Han and S. Zhan, J. Hazard. Mater., 2017, 338, 33.

15 L. Tang, Y. Fang, Y. Pang, G. M. Zeng, J. J. Wang, Y. Y. Zhou, Y. C. Deng, G. D. Yang, Y. Cai and J. Chen, Chem. Eng. J., 2014, 254, 302.

16 W. Tanglumlert, T. Imae, T. J. White and S. Wongkasemjit, Mater. Lett., 2008, 62, 4545.

17 J. E. Chen, H.-Y. Lian, S. Duttacec, S. M. Alshehri, Y. Yamauchi, M. T. Nguyen, T. Yonezawa and K. C.-W. Wu, Phys. Chem. Chem. Phys., 2015, 17, 27653.

18 J. C. Yan, W. G. Gao, M. G. Dong, L. Han, L. B. Qian, C. P. Nathanail and M. F. Chen, Chem. Eng. J., 2016, 295, 309.

19 B. Sahoo, S. K. Sahu, S. Nayak, D. Dhara and P. Pramanik, Catal. Sci. Technol., 2012, 2, 1367.

20 N. Kato and N. Kato, Microporous Mesoporous Mater., 2016, 219, 230.

21 T. A. Arica, E. Ayas and M. Y. Arica, Microporous Mesoporous Mater., 2017, 243, 164.

22 C. Wang, S. Y. Tao and C. G. Meng, J. Mater. Chem., 2012, 22, 7179.

23 S. Y. Tao, Z. Y. Zhu, C. G. Meng and C. Wang, Microporous Mesoporous Mater., 2013, 171, 94.

24 B. K. Vashisth, J. S. Bangruwa, A. Beniwal, S. P. Gairola, A. Kumar, N. Singh and V. Verma, J. Alloys Compd., 2017, 698, 699. 
25 G. P. Anipsitakis and D. D. Dionysiou, Environ. Sci. Technol., 2003, 37, 4790.

26 J. Fernandez, P. Maruthamuthu, A. Renken and J. Kiwi, Appl. Catal., B, 2004, 49, 207.

27 H. L. Xie, W. He and G. Chen, J. Chin. Chem. Soc., 2015, 62, 1144.

28 H. L. Xie and T. Y. Zhang, Int. J. Nanosci., 2015, 13, 1460010.

29 S. Gomez, L. Lerici, C. Saux, A. L. Perez, C. D. Brondino, L. Pierella and L. Pizzio, Appl. Catal., B, 2017, 202, 580.

30 P. Schmidt-Winkel, W. W. Lukens, D. Y. Zhao, P. D. Yang, B. F. Chemlka and G. D. Stucky, J. Am. Chem. Soc., 1999, 121, 254.

31 J. E. Chen, H.-Y. Lian, S. Duttacec, S. M. Alshehri, Y. Yamauchi, M. T. Nguyen, T. Yonezawa and K. C.-W. Wu, Phys. Chem. Chem. Phys., 2015, 17, 27653.

32 S. Liu, H. Y. Zhang, X. J. Meng, Y. L. Zhang, L. M. Ren, F. Nawaz, J. Y. Liu, Z. Q. Li and F.-S. Xiao, Microporous Mesoporous Mater., 2010, 136, 126.

33 M. A. Betiha, A. M. Rabie, A. M. Elfadly and F. Z. Yehia, Microporous Mesoporous Mater., 2016, 222, 44.

34 Y. X. Wang, L. Zhou, X. G. Duan, H. Q. Sun, E. L. Tin, W. Q. Jin and S. B. Wang, Catal. Today, 2015, 258, 576.

35 M. Y. Wei, L. Gao, J. Li, J. Fang, W. X. Cai, X. X. Li and A. Xu, J. Hazard. Mater., 2016, 316, 60.

36 J. Conradie and E. Erasmus, Polyhedron, 2016, 119, 142.

37 C. Prasad, K. Sreenivasulu, S. Gangadhara and P. Venkateswarlu, J. Alloys Compd., 2017, 700, 252.

38 J. Liu, J. H. Zhou, Z. X. Ding, Z. W. Zhao, X. Xu and Z. D. Fang, Ultrason. Sonochem., 2017, 34, 953.

39 G. Nie, J. Huang, Y. Z. Hu, Y. B. Ding, X. Y. Han and H. Q. Tang, Chin. J. Catal., 2017, 38, 227.

40 Y. J. Yao, Y. M. Cai, G. D. Wu, F. Y. Wei, X. Y. Li, H. Chen and S. B. Wang, J. Hazard. Mater., 2015, 296, 128.

41 N. Y. He, C. Yang, P. F. Xiao, G. H. Wang, Y. J. Zhao, Z. H. Lu and C. W. Yuan, Stud. Surf. Sci. Catal., 2000, 129, 483.

42 J. Conradie and E. Erasmus, Polyhedron, 2016, 119, 142-150. 43 H. Z. Liu, T. A. Bruton, W. Li, J. V. Buren, C. Prasse, F. M. Doyle and D. L. Sedlak, Environ. Sci. Technol., 2016, 50, 890-898.

44 C. Zhu, G. Fang, D. D. Dionysiou, C. Liu, J. Gao, W. Qin and D. Zhou, J. Hazard. Mater., 2016, 316, 232-241.

45 J. Deng, S. F. Feng, X. Ma, C. Tan, H. Wang, S. Zhou, T. Zhang and J. Li, Sep. Purif. Technol., 2016, 167, 181-189.

46 C.-H. Tsai, W.-C. Chang, D. Saikia, C.-E. Wu and H.-M. Kao, J. Hazard. Mater., 2016, 309, 236.

47 L. X. Hu, F. Yang, W. C. Lu, Y. Hao and H. Yuan, Appl. Catal., $B, 2013$, 134-135, 7.

48 Y. B. Wang, X. Zhao, D. Cao, Y. Wang and Y. F. Zhu, Appl. Catal., B, 2017, 211, 79.
49 C.-H. Tsai, W.-C. Chang, D. Saikia, C.-E. Wu and H.-M. Kao, J. Hazard. Mater., 2016, 309, 236.

50 S. Larouk, R. Ouargli, D. Shahidi, L. Olhund, T. C. Shiao, N. Chergui, T. Sehili, R. Roy and A. Azzouz, Chemosphere, 2017, 168, 1648.

51 Z. C. Bian, X. Meng, M. Tao, Y. H. Lv and Z. Xin, Fuel, 2016, 179, 193.

52 S. N. Azizi, S. Ghasemi, A. Samadi-Maybodi and M. RanjbarAzad, Sens. Actuators, B, 2015, 216, 271.

53 Y. L. Dong, X. L. Zhan, X. Y. Niu, J. Li, F. L. Yuan, Y. J. Zhu and H. G. Fu, Microporous Mesoporous Mater., 2014, 185, 97.

54 A. T. Shah, M. I. Din, F. N. Kanwal and M. L. Mirza, Arabian J. Chem., 2015, 8, 579.

55 S. N. Azizi, S. Ghasemi, A. Samadi-Maybodi and M. RanjbarAzad, Sens. Actuators, B, 2015, 216, 271.

56 D. Y. Zhao, J. L. Feng, Q. S. Huo, N. Melosh, G. H. Fredrickson, B. F. Chmelka and G. D. Stucky, Science, 1998, 279, 548.

57 W. R. Chen, X. K. Li, Z. Q. Pan, S. S. Ma and L. S. Li, Sep. Purif. Technol., 2017, 173, 99.

58 S. Karthikeyan, M. P. Pachamuthu, M. A. Isaacs, S. Kumar, A. F. Lee and G. Sekaran, Appl. Catal., B, 2016, 199, 323.

59 V. R. Elías, G. O. Ferrero, R. G. Oliveira and G. A. Eimer, Microporous Mesoporous Mater., 2016, 236, 218.

60 C. Q. Tan, N. Y. Gao, Y. Deng, J. Deng, S. Q. Zhou, J. Li and X. Y. Xin, J. Hazard. Mater., 2014, 276, 452.

61 X. H. Li, W. L. Guo, Z. H. Liu, R. Q. Wang and H. Liu, Appl. Surf. Sci., 2016, 369, 130.

62 X. Y. Chen, X. L. Qiao, D. G. Wang, J. Lin and J. W. Chen, Chemosphere, 2007, 67, 802.

63 C. Cai, Z. Zhang and H. Zhang, J. Hazard. Mater., 2016, 313, 209.

64 E. Saputra, S. Muhammad, H. Q Sun, H.-M. Ang, M. O. Tadé and S. B. Wang, Appl. Catal., B, 2013, 142-143, 729.

65 J. K. Du, J. G. Bao, X. Y. Fu, C. H. Lu and S. H. Kim, Appl. Catal., B, 2016, 184, 132.

66 I. Mazilu, C. Ciotonea, A. Chirieac, B. Dragoi, C. Catrinescu, A. Ungureanu, S. Petit, S. Royer and E. Dumitriu, Microporous Mesoporous Mater., 2017, 241, 326.

67 M. J. Huang, T. Zhou, X. H. Wu and J. Mao, Water Res., 2017, 119, 47.

68 A. Ghauch, G. Ayoub and S. Naim, Chem. Eng. J., 2013, 228, 1168.

69 L. Tang, Z. H. Xie, G. M. Zeng, H. R. Dong, C. Z. Fan, Y. Y. Zhou, J. J. Wang, Y. C. Deng, J. J. Wang and X. Wei, RSC Adv., 2016, 6, 25724.

70 L. Luo, A. Zhang, M. J. Janik, K. Li, C. Song and X. Guo, Appl. Surf. Sci., 2017, 396, 78. 